$( \pm 3.2)$ years in cases of SSc-related death, as compared to $18.3( \pm 11.0)$ years in deaths not related to SSC.

Conclusions: SSc prevalence $\left(13,8 / 10^{5}\right)$ in Crete is consistent with previous (2002) Greek studies $\left(15,4 / 10^{5}\right)^{4}$ albeit lower compared to other European countries and USA ${ }^{5}$. Despite its rarity, the disease has significant burden. The SSc-related mortality occurs early in the disease course. Early recognition and treatment may decrease disease burden and improve the outcomes.

References:

[1] Chifflot H, et al Semin Arthritis Rheum. 2008 Feb;37(4):223-35.

[2] Maricq HR, et al Arthritis Rheum. 1989 Aug:32(8):998-1006.

[3] M. Elhai, C. et al Rheumatology (2012); 51(6):1017-1026.

[4] Alamanos et al Semin Arthritis Rheum. 2005 Apr;34(5):714-20.

[5] Mayes MD, Rheum Dis Clin North Am. 2003 May;29(2):239-54.

Disclosure of Interest: None declared

DOI: 10.1136/annrheumdis-2017-eular.2847

\section{SAT0331 AMINAPHTONE INCREASES SKIN BLOOD PERFUSION AND IMPROVES CLINICAL SYMPTOMS IN PATIENTS WITH BOTH PRIMARY AND SECONDARY RAYNAUD'S PHENOMENON: AN OPEN SIX MONTH PILOT STUDY}

B. Ruaro, S. Paolino, C. Pizzorni, E. Alessandri, E. Gotelli, M. Cutolo, A. Sulli. Research Laboratory and Academic Division of Clinical Rheumatology, Department of Internal Medicine, University of Genova, IRCCS AOU San Martino, Genoa, Italy

Background: Aminaphtone (1,4-Dihydroxy-3-methyl-2-naphthyl-4-aminobenzoate) is a vasoactive drug that was recently demonstrated to improve the symptoms of Raynaud's phenomenon (RP) and to down-regulate endothelin-1 production by endothelial cells $(1-3)$.

Objectives: To evaluate skin blood perfusion and clinical symptom changes during aminaphtone treatment in patients with both primary and secondary RP, during a six-month follow-up.

Methods: Forty-six patients with active RP were enrolled during routine clinical assessment in November 2015 (11 primary RP, mean age 49 \pm 19 SD years, mean RP duration $6 \pm 3$ years; 35 secondary RP to systemic sclerosis, mean age $61 \pm 17$ years, mean RP duration $11 \pm 9$ years), after informed consent. Aminaphtone was administered $75 \mathrm{mg}$ twice daily (off label) in addition to current treatments (the patients were on a stable drug regimen for at least two months before, which remained unmodified during the follow-up). Blood perfusion was measured by Laser Speckle Contrast Analysis (LASCA) and values recorded as perfusion units (PU) (4), at the level of fingertips, periungual areas, dorsum and palm of hands, and face, at baseline (T0), after one (T1), four (T4), twelve (T12) and twenty-four (T24) weeks of treatment. Raynaud's condition score (RCS) and both frequency and duration of Raynaud's attacks were assessed at the same time. Forty-six patients with RP (9 primary RP and 37 secondary RP to systemic sclerosis) not treated with aminaphtone were also enrolled as a control group and evaluated at T0 and T24.

Results: A progressive statistically significant increase of blood perfusion was observed from T0 to T12 in all skin areas analyzed (median PU at T0, T1, T4, T12, T24 respectively: fingertips $55,88,101,107,98$ periungual areas 44,88 , $91,92,92$; dorsum of hands $38,61,71,75,75$; palm of hands $56,85,89,94$, 82 ; whole face $127,138,144,159,129 ; \mathrm{p}<0.001$ for all areas). From $\mathrm{T} 12$ to T24 was not observed any further increase of blood perfusion. A progressive statistically significant decrease of RCS (median at T0, T1, T4, T12, T24: 7, 6, $4,4,4 ; p<0.0001$ ), frequency of Raynaud attacks/day (median: 2, 2, 1, 1, 1; $p<0.0001$ ) and Raynaud duration (median: 20, 20, 10, 4, 4 minutes; $p<0.0001$ ) was also recorded from T0 to T12. The results were similar in both primary and secondary RP patients $(p=0.40)$. Aminaphtone administration had to be stopped in 2 patients due to headache, and one patient was lost during follow-up. Any statistically significant variation of blood perfusion was not observed in the control group (median PU at T0 and T24 respectively: fingertips 70, 71; periungual areas 68,70 ; dorsum of hands 57,57 ; palm of hands 59,59 ; whole face 132,130 ; $\mathrm{p}=\mathrm{n} . \mathrm{s}$. for all areas)

Conclusions: This study demonstrates that aminaphtone treatment seems able to increase skin blood perfusion and to improve RP symptoms, even in patients affected by systemic sclerosis. These preliminary results should be further confirmed by a randomized clinical trial, also to assess the role that aminaphtone plays in the treatment/prevention of disease clinical complications.

References:

[1] Parisi S, et al. Am J Int Med 2015;3;204-9.

[2] Scorza R, et al. Drugs R D 2008:9:251-7.

[3] Salazar G, et al. Eur J Pharmacol. 2016;782:59-69.

[4] Ruaro B, et al. Ann Rheum Dis. 2014;73:1181-5.

Disclosure of Interest: None declared

DOI: 10.1136/annrheumdis-2017-eular.4470

\section{SAT0332 HEPATOBILIARY INVOLVEMENT IN SYSTEMIC SCLEROSIS AND THE CUTANEOUS SUBSETS. CHARACTERISTICS AND SURVIVAL OF PATIENTS FROM THE SPANISH RESCLE REGISTRY}

B. Marí Alfonso ${ }^{1}$, C. Tolosa-Vilella ${ }^{2}$, L. Morera-Morales ${ }^{2}$, A. Marín-Ballvé ${ }^{3}$ J. Chamorro Fernández ${ }^{4}$, X. Pla Salas ${ }^{5}$, B. Madroñero-Vuelta: ${ }^{6}$, C. Simeón Aznar ${ }^{7}$ on behalf of RESCLE Investigators of the Autoimmune Diseases Study Group (GEAS). ${ }^{1}$ Internal Medicine, Corporació Sanitària Parc Tauli; ${ }^{2}$ Internal Medicine, Corporació Sanitària Parc Tauli. Sabadell. Barcelona, Sabadell; ${ }^{3}$ Internal Medicine, Hospital Clínico Universitario Lozano Blesa, Zaragoza; ${ }^{4}$ Internal Medicine, Complejo Asistencial Universitario de Salamanca,

Salamanca; ${ }^{5}$ Internal Medicine, Consorci Hospitalari de Vic, Barcelona; ${ }^{6}$ Internal Medicine, Hospital General San Jorge, Huesca; ${ }^{7}$ Internal Medicine, Hospital Universitario Vall d'Hebron, Barcelona, Spain

Background: Hepatobiliary involvement (HBI) has been associated to systemic sclerosis (SSc). However, it is not considered as characteristic clinical manifestation of that autoimmune disease

Objectives: To assess the prevalence and causes of HBI in SSc and to investigate the clinical characteristics and prognosis of SSc patients with HBI (SSc-HBI) and without $\mathrm{HBI}$ (SSc-non $\mathrm{HBI}$ ).

Methods: Up to January 2015, 1572 SSc patients were collected in the Registro de ESCLErodermia (RESCLE) and causes of hepatobiliary disturbances were recorded. We investigated the $\mathrm{HBI}$ related characteristics and survival from the entire cohort and according to the following cutaneous subsets: dcSSc, IcSSc, and SSc sine scleroderma (SSSSc).

Results: Out of 1572, $118(7.5 \%)$ patients had $\mathrm{HBI}$, and primary biliary cholangitis (PBC) was largely the main cause $(n=67,4.3 \%)$, followed by autoimmune hepatitis $(n=19,1.2 \%)$, and anti-mitochondrial negative PBC $(n=6,0.4 \%)$. Other causes of HBI were: secondary liver diseases $(n=11,0.7 \%)$, SSc-related $\mathrm{HBI}(n=7,0.4 \%)$, nodular regenerative hyperplasia $(n=3,0.2 \%)$, liver cirrhosis $(n=3,0.2 \%)$, and unknown origin $(n=2,0.1 \%)$. In multivariate analysis, HBI was independently associated to lesser risk of dcSSc $(5.1 \%$ vs $24.4 \%$, OR: $0.18, p=0.005 \%)$, and higher frequency of calcinosis ( $26 \%$ vs $18 \%$, OR: $1.80 ; p=0.028)$, left ventricular diastolic dysfunction ( $46 \%$ vs $27 \%$, OR: $1.73 ; p=0.027)$, sicca syndrome $(51 \%$ vs $29 \%$, OR: $2.03 ; p=0.003$ ), and anti-centromere antibodies (ACA, $73 \%$ vs $44 \%$, OR: $1.86 ; p=0.023)$. According to the cutaneous subsets, HBI was associated: (1) in IcSSc subset, to longer time from SSc onset to diagnosis $(10.8 \pm 12.5$ vs $7.2 \pm 9.3$, OR: $1.03 ; p=0.012$ ), sicca syndrome ( $54 \%$ vs $33 \%$, OR: $1.96 ; p<0.001$ ), and ACA ( $80 \%$ vs $56 \%$, OR: $2.64 ; p<0.001$ ); and (2) in ssSSc subset, to sicca syndrome (44\% vs $19 \%$, OR: $3.43 ; p=0.018$ ). No associations were found in dcSSc subset. $\mathrm{HBI}$ was the cause of death in $2.3 \%$ patients. Kaplan-Meier cumulative survival for the SSc cohort and the cutaneous subsets, according to the presence or absence of HBI showed no differences.

Conclusions: The prevalence of HBI in SSc was 7.5\%. Primary autoimmune liver diseases accounted for $77 \%$ of all conditions. PBC was the main cause of $\mathrm{HBI}$ reaching $4.6 \%$ of the overall SSc cohort. Patients with $\mathrm{HBI}$ were rarely classified as dcSSc subset and the elapsed time from the first SSc symptom to SSc diagnosis was longer. Calcinosis cutis, diastolic dysfunction, sicca syndrome, and the presence of ACA were all independently associated to HBI. In IcSSc subset, HBI was associated to sicca syndrome and ACA but in ssSSc only sicca syndrome was more prevalent. HBI was the cause of death in $2.3 \%$

References:

[1] Assassi S., Fritzler MJ, Arnett FC, et al., Primary biliary cirrhosis (PBC), PBC autoantibodies, and hepatic parameter abnormalities in a large population of systemic sclerosis patients. J Rheum 2009; 36: 2250-2256.

[2] Rigamonti C, Shand LM, Feudjo M, et al. Clinical features and prognosis of primary biliary cirrhosis associated with systemic sclerosis. Gut 2006; 55; 388-394.

Disclosure of Interest: None declared

DOI: 10.1136/annrheumdis-2017-eular.5745

\section{SAT0333 PREVALENCE OF SARCOPENIA IN PATIENTS WITH SYSTEMIC SCLEROSIS ACCORDING TO THE REVISED CRITERIA OF THE EUROPEAN WORKING GROUP ON SARCOPENIA IN OLDER PEOPLE}

C. March ${ }^{1}$, E. Siegert ${ }^{1}$, E. Preis ${ }^{1}$, A. Makowka ${ }^{1}$, F. Buttgereit ${ }^{1}$,

G. Riemekasten ${ }^{2}, \mathrm{~K}$. Norman ${ }^{3} .{ }^{1}$ Department of Rheumatology and Clinical Immunology, Charité-Universitätsmedizin Berlin, Berlin; ${ }^{2}$ Department of

Rheumatology, University of Luebeck, Luebeck; ${ }^{3}$ Research Group on Geriatrics, Charité-Universitätsmedizin Berlin, Berlin, Germany

Background: Systemic Sclerosis (SSc) is a chronic inflammatory connective tissue disease that is often associated with gastrointestinal involvement and myopathy. Sarcopenia is defined as age-associated loss of muscle mass, strength and function with profound impact on functionality as well as on mortality (1). "Secondary sarcopenia" has now been described in the context of severe and chronic disease such as malignant disease or inflammatory disorders (2) and been linked to poor clinical outcome.

Objectives: Analysis of the prevalence of sarcopenia in patients with SSc with respect to clinical characteristics, quality of life and severity of physical impairment. Methods: 129 patients were included. Sarcopenia was defined according to the 